\title{
Method and approaches to provide feedback from nuclear and covariance data adjustment for improvement of nuclear data files: Major findings of the NEA WPEC Subgroup 39
}

\author{
Massimo Salvatores ${ }^{1}$ and Giuseppe Palmiotti ${ }^{2}$ \\ ${ }^{1}$ Scientific A dvisor, Reactor and Fuel Cycle, France \\ 2 INL, USA
}

\begin{abstract}
Nuclear data adjustments using integral experiments play since several decades a crucial role in providing reactor designers and fuel cycle analysts with nuclear data with reduced and validated uncertainties in order to allow design optimization while meeting safety and economics requirements. A djustment methods, choice of integral experiments and covariance data assessment should be carefully defined in order to produce credible and physically acceptable adjustments, applicable to a wide range of systems. The OECD-NEA has established a series of Expert Groups since 2005 to investigate the key scientific issues and to provide recommendations for applications. The present paper discusses the major results of the most recent of these Expert G roups together with an indication of the path forward.
\end{abstract}

\section{Introduction and background}

The OECD-NEA WPEC Subgroup 33 final report issued in 2014 [1] indicated that a deeper understanding of the methodologies and of their applications implies that cross section adjustments can provide crucial feedback to evaluators and differential measurement experimentalists to improve the knowledge of neutron cross sections to be used in a wide range of applications.

This new role for cross section adjustment requires to solve a new series of issues: definition of criteria to assess the reliability and robustness of an adjustment; requisites to assure the quantitative validity of the covariance data; criteria to identify inconsistency between differential and integral data; and definitions for consistent approaches in the use of both adjusted data and a posteriori covariance data to improve quantitatively nuclear data files.

It is also crucial to provide methods and define conditions to generalize the results of an adjustment in order to evaluate the "extrapolability" of the results of an adjustment to a different range of applications (e.g., different reactor systems) for which the adjustment was not initially intended and to suggest guidelines to enlarge the experimental data base in order to meet needs that were identified by the cross section adjustment.

A key objective of the subsequent Subgroup 39 has then been to investigate criteria and practical approaches to effectively utilize the results of sensitivity analyses and cross section adjustments to provide feedback to eval uators and differential measurement experimentalists, and ultimately to improve the knowledge of neutron cross sections, uncertainties, and correlations to be used in reactor and fuel cycle applications.
W ith the availability of new covariance data, it became urgent to revisit the fundamental question of adjustment trend reliability. Stress tests did point out potential inconsistencies if the integral data base was not carefully investigated and the uncertainties, potential systematic errors, and correlations were not documented.

The Subgroup did tackle the key issues of investigating methods to make the adjustment approach more robust, in particular to avoid compensations when modifications (i.e. adjustments) of cross sections of different isotope reactions were suggested.

The criteria in the selection of integral experiments were reviewed and new priorities were suggested. Finally, the role of covariance data, their reliability and completeness were discussed.

Moreover "a-posteriori" covariance data utilization should still be investigated in order to get the maximum benefit from an adjustment study.

\section{The framework}

The central role of nuclear data in reactor physics can be summarized as shown in the following scheme:

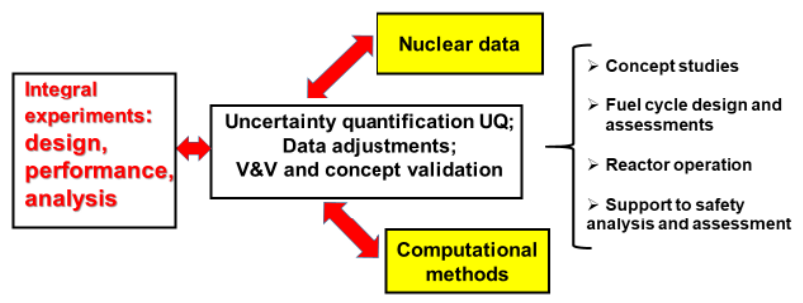

Fig. 1. Nuclear data in the frame of reactor physics.

\footnotetext{
Corresponding author: salvatoresmassimo@orange.fr
} 
N uclear Data needs assessment, U Q , and adjustments have been recognized as a key area for research in the nuclear energy domain. The NEA W PEC launched a series of initiatives: The starting point $(\sim 2005)$ has been a data needs assessment for GENERATION-IV, ADS and new nuclear fuel cycles with waste management issues. Consequently a first list of data priorities for GEN -IV and ADS reactors was established within SG26 [2] and implemented in the HPRL (High Priority Request List) at NEA .

The next step did tackle data adjustment and covariance data comparison (Subgroup 33) and a comprehensive compilation of methods and a benchmark exercise were performed to understand if adjustments converge, starting from different $x$-section data bases and using different covariance data [3].

Successively, reliability issues were at the heart of Subgroup 39 mandate. K ey issues were pointed out:

- How to make adjustment more reliable and avoid compensation effects. Stress tests and innovative adjustment methods/strategies were developed $[4,5,6,7]$.

- $\mathrm{N}$ ew approaches were also suggested towards a sound integral data selection to be used in an adjustment $[8,9]$.

Findings and recommendations in three major areas of investigation, i.e. adjustments, integral experiments and covariance data, will be summarized in the following paragraphs.

\section{Adjustment methods}

\subsection{How to adjustments}

The methods are well understood and comparable [10]. However, different perceptions of their role:

Use "as such" in design and safety assessments applied to actual reactor design (as it has been the case e.g. of SUPERPHENIX [11]).

U se as guidelines for improving evaluations.

Use directly in evaluation (the case of ${ }^{239} \mathrm{Pu}$, using JEZEBEL experiments [12] or more recently [13].

Use to modify "online" evaluated files e.g. continuous energy adjustment [14] or nuclear model parameters adjustment (e.g. the consistent method [15]) which is potentially the most powerful approach

\subsection{Adjustments: multigroup, continuous energy or model parameters?}

If adjustments are used "as such", the multigroup choice is appropriate, but should be consistent with design methods. In other terms, the analysis of the integral experiments and the design calculations should be made with the same codes, approximations, if any, and multigroup structures.

When adjustments are used to suggest evaluation modification guidelines, a reduced number of energy "bands", chosen according to physics criteria, can be a better choice [16].

If adjustments are used to directly improve evaluated nuclear data files, a continuous energy adjustment [14] is probably the best choice. However, the most "physics driven" approach is an adjustment of the reaction model parameters that enters into an evaluation (e.g. the consistent method [15] and work done at CEA - Cadarach [17])

The consistent method makes use of the sensitivities of integral experiments to model parameters $\mathbf{p} \mathbf{k}$ :

$$
\frac{\Delta R}{\Delta p_{k}}=\sum_{j} \frac{\Delta R}{\Delta \sigma_{j}} \times \frac{\Delta R \sigma_{j}}{\Delta p_{k}}
$$

where $\mathbf{R}$ is an integral parameter and $\boldsymbol{\sigma}_{\mathrm{j}}$ a multigroup cross section (the $\mathrm{j}$ index accounts for isotope, cross section type and energy group) and $\mathbf{p}_{k}$ are the model parameters.

Exploratory studies performed in Ref.[18] indicate both the potential of the method and issues still to be solved.

\subsection{Compensation effects}

The problem of compensations in cross section adjustments was illustrated e.g. in Ref. [19]. In fact, in many cases the adjustment can produce untrustworthy results in terms of adjusted cross sections, when possible a-priori forms of compensation exist. Examples, among others, of source of compensations are:

- $V$ ariations of different reactions of the same isotope can compensate each other (e. g. ${ }^{239} \mathrm{Pu}$ fission spectrum $\chi$ and inelastic cross section)

- Different isotope cross section variations have opposite and compensating effects (e.g. ${ }^{238} \mathrm{U}$ capture increase associated to ${ }^{239} \mathrm{Pu}$ fission increase)

These potential sources of compensations can produce unreliable adjustments if there is lack of specific reactions and of cross correlations in the covariance matrix or if there are inadequate values in the covariance matrix that in an adjustment lead adjusting certain cross sections more than others, e.g. due to unjustified very small uncertainty values. A major improvement in order to cope with the problem of compensations, regarding the availability of integral experiments, is to use more integral experiments of the elemental type (see paragraph 4) that allow to discriminate among the parameters (cross sections), and, therefore, to insure the reliability of the adjustment. In particular there is a need for specific (preferably of elemental type) integral experiments:

Along this line we define, in the following, an adjustment strategy, PIA (Progressive Incremental Adjustment [5]) that takes advantage of an ampler availability of integral experiments of the elemental type in order to limit the effect of compensations.

In the proposed PIA method, the starting point is giving priority to the utilization of experiments of elemental type (those sensitive to a specific cross section), following a definite hierarchy on which type of experiment to use. Once an adjustment step is performed, both the new adjusted data and the new covariance matrix are kept. This limits the range of variability of the adjusted cross sections. In the final steps integral experiments that are sensitive to a large variety of cross sections (global type like critical mass) are added. Ref. [6].

Similar methods have also been developed, e.g. see

A different approach has been proposed in Ref. [20]. In fact, one can detect the compensation effects by understanding the adjustment mechanism. For this purpose, 
three indices have been proposed [20]: Mobility in adjustment, Adjustment motive force, Adjustment potential and their investigation can provide indication on the physics validity of the adjustments.

Finally, stress tests have been used as a tool for detecting compensation phenomena [4].

As an example, to understand how compensation effects can arise in nuclear data adjustments, a stress test on ${ }^{235} \mathrm{U}(\mathrm{n}, \mathrm{f})$ cross sections has been performed using $\mathrm{U}-\mathrm{Pu}$ fueled fast critical experiments together with a set of integral benchmark information specifically sensitive to ${ }^{235} \mathrm{U}$ data. The test results show that the a-posteriori values of integral and differential data can change from case to case when the input integral information changes. This indicate that the choice of the right constraints (i.e. the appropriate integral experiments) is a key feature of any adjustment.

\section{Integral experiments}

M ost of the Subgroup 39 findings, point out to the selection of appropriate experiments providing selective information on inelastic, elastic, $(n, 2 n)$, fission, and capture data. Finally, integral experiment uncertainties and correlations should be carefully assessed, as previous studies have demonstrated [3]. In particular, any possible source of systematic errors should be investigated, and practical methods to detect them have been proposed [3].

a) Separated isotope sample irradiation experiments (for capture and $(n, 2 n)$ cross section adjustments)

b) Experiments with enhanced sensitivity in a specific energy range, e.g. irradiation experiments with enhanced capture data sensitivity in the range from e.g. few hundred $\mathrm{eV}$ to $\mathrm{leV}$ and with appropriate filters to tune the spectrum at the irradiation position;

c) Spectrum indices (information on capture and fission and, at a lesser extent, inelastic)

d) "Flat" or "steep" adjoint flux reactivity experiments (to separate inelastic from absorption cross section effects and, partly, from fission spectrum reactivity effects)

e) Experiments with systematic variation e.g. of the spectrum hardness and with and w/o coolant for selective spectrum effects

f) Oscillation experiments to get the reactivity of single isotope samples in different spectra

g) Reactivity/atom values of e.g. fissile isotopes. This has been done see e.g. R ef. [21] using experiments where, starting from a reference critical configuration, one did replace it (e.g. at the center of the zero-power reactor) with a new composition that differed from the reference by a known amount of one isotope. The measurement of the new reactivity with respect to the critical reference, gives a direct information on the reactivity of that specific isotope:

$$
\Delta \rho^{p}=V^{p} / V^{R E F} \rho_{i}\left(N_{i}^{p}-N_{i}^{R E F}\right)
$$

$\mathrm{i}$ index of individual isotope. $V_{p}$ volume of the central substitution zone, $V_{\text {REF }}$ volume of the central reference zone. $N_{i}^{p}-N_{i}^{R E F}$ is the variation of the number of atoms of isotope $i$ associated to substitution "p"

h) N eutron transmission or leakage experiments (mostly for inelastic and elastic cross sections and for angular scattering effects)

i) Reaction rate spatial distribution slopes (elastic, and inelastic, including, partly, angular scattering effects)

l)Effective delayed neutron fraction (ßeff) measurements, providing information on fission, prompt neutron spectrum and inelastic cross section competition, complementary to spectrum indices and criticality measurements

As far as new experiments, most historical facilities are no more available. One should consider the possibility to get even limited access to the new experimental reactors that are being launched (e.g. the extremely well-equipped reactor $M B I R$ ) and to make use of simple facilities like NRAD at INL, where physics experiments could be performed with limited cost and resources [22].

Finally, the transformation of existing thermal neutron facilities in coupled fast-thermal neutron spectrum systems [23], can offer a further source of physics experiments, as it has been done successfully in the past [24].

\section{Covariance data}

In the last two decades, and partly thanks to the WPEC pioneer, and provocative, work of Subgroup 26[2], there has been an important international activity aiming to the evaluation of covariance data. The results of the research performed within Subgroup 33 has shown [1] that the use of a specific covariance data set in $U Q$ has strong impact on concept feasibility, safety assessments, optimization in design and operation, fuel cycle features etc. M oreover, it has been shown that adjustments very much depend on apriori uncertainties and correlations. Reliability (the case of some very low uncertainties on key data, like fission cross sections) and completeness of covariance data sets (e.g. angular distributions, gamma production data) are still key issues: covariance data evaluation methods are still to be explored according to a recommendation of the Subgroup 39. Moreover, it has been pointed out that the covariance data evaluation should al so provide consistency with new needs, as it will be discussed in 5.1. Finally, it has been pointed out that, when adjustments are performed, the use of a-posteriori correlations[25, 26] presents a number of issues that have to be fully understood, in particular when extrapolating the results of an adjustment beyond the system domain originally defined for application of the adjustment itself (see 5.2).

\subsection{Covariance data and target accuracies}

$N$ ew emerging needs require not only data evaluation but covariance data consolidation. Potential industry driven needs:

- Reactivity coefficients when M OX-loaded LWR with tight lattices or with multirecycle. The role of uncertainties is crucial to assess safety related composition limitations.

The use of a-posteriori correlations is often limited to the cross-section covariance matrix diagonal elements. Little is done with the new correlations (i.e. off diagonal elements) and usually there is no use of the cross correlations between integral experiments and cross 
sections, shown in the scheme above. However, this situation is fairly unsatisfactory, since an important contribution to any uncertainty reduction, comes from induced correlations and cross correlations.

- New materials: cladding, coatings: Si, Al etc (ATF, EATF, Fusion) data and uncertainties

- SM R and longer burn-up challenges

- Generalized BU increase

- In general for most advanced concept assessment beyond a first exploratory phase

Moreover, data and covariance needs could also become crucial for specific issues related to innovative fuel cycles. In fact, if Accelerator Driven Systems ADS (or burner $F R$ or even fusion-fission hybrids, FFH) are considered for waste management e.g. with full $M A$ recycle, in particular with metal fuels, they can offer a real breakthrough, R ef.27. In that case, new cross section and covariance data will be necessary to allow the feasibility assessment of any associated fuel cycle strategy. Also, if the Th cycle becomes a serious option, e.g. associated to molten salts reactors, much improved cross sections and covariance data both for the reactor and the fuel cycle will be needed. Finally, some decommissioning issues will benefit from a better nuclear data knowledge.

A ll these potential needs require:

New target uncertainties. The last coordinated international assessment is more than 15 years old and it was performed in a very different environment, mostly associated to the emergence of GENERATION-IV initiative.

An update of High Priority Request List (HPRL) at the NEA DataB ank to define new priorities for evaluation and experiments. When new target accuracies will have been assessed, a revision of adjustment and bias factor methods could be necessary.

The Subgroup 39 recommendation has been to take the target accuracy issue as a key objective of a new WPEC expert group in this domain (SG46 on "Efficient and Effective Use of Integral Experiments for Nuclear Data Validation").

\subsection{A-posteriori correlations}

One issue, pointed out by Subgroup 39, that deserves further investigation is the understanding of how to exploit induced (i.e. a-posteriori) correlations between nuclear data and experiments.

\section{Conclusions}

The NEA Working Party on Evaluation Cooperation has supported successfully a continuous activity during the last two decades in the field of the use of integral experiments to provide feedback to evaluation research. A large international participation has succeeded in defining scientific issues, providing recommendations and promoting new developments. The findings of the most recent Expert Group, Subgroup 39, can be summarized as follows:

More robustness and "physics" have been achieved in adjustment techniques with new theoretical developments.
As for the key challenge to avoid compensations, several new methods are becoming available and applicable in current adjustment exercises.

As for the role and the type of integral experiments, new paradigms have been indicated:

- The choice of representative experiments is a priority. This should be done with analytical tools based on sensitivity analysis

- Experiments for single (elemental) physics effects should be given priority. M any experiments of that type have been performed in the past but not always fully exploited

- "Global" experiments (e.g. keff) should be mostly used as a-posteriori validation, to avoid compensations and ambiguities

- The use of integral experiments directly in evaluations, should be carefully discussed, since they could hide the potential double use of the same experiments (i.e. once in evaluation and once in the validation process).

A new SG46: "Efficient and Effective Use of Integral Experiments for Nuclear Data Validation" has been launched to tackle the recommendations of Subgroup 39 in order to:

- Consolidate the adjustment techniques improvement;

- To promote further studies on covariance data reliability and completeness, in conjunction with SG 44;

- A new activity has been defined with high priority, i.e. the assessment of revised cross section target accuracies for an expanded number of reactor and fuel cycle systems (including M SR, ADS, SM R, Pu recycle etc), according to revised design target accuracies. This new activity should provide updated input to HPRL

- The Subgroup 39 activity has stressed the effectiveness of international collaboration within NEA and has facilitate a closer and very fruitful link between Reactor and Nuclear Physics communities.

\section{Acknowledgements}

The authors gratefully acknowledge the outstanding contributions of all the members of Subgroup 39 and the invaluable technical secretariat support of 0 . Cabellos and M. Fleming of OECD-NEA.

\section{References}

1. M. Salvatores et al., Nucl. Data Sheets, 118, 38-71 (A pril 2014)

2. G.Aliberti, G.Palmiotti and M. Salvatores, A nnals of Nucl. Energy, 33, 700-733 (2006)

3. G. Palmiotti, M. Salvatores et al., Nucl. Data Sheets, 118, 596-636 (2014)

4. H. W u, Y. Qin and M. Salvatores, EPJ 146, 06027 (2017)

5. G. Palmiotti, M. Salvatores, M \& C 16-20 A pril 2017, Jeju, Korea.

6. Pelloni S, Rochman D, A nnals of Nucl. Eng. 129, 79 (2019)

7. K. Y okoyama andT. K itada, J. of N ucl. Sci. and Tech., 56, 87-104 (2019)

8. M. Salvatores, G. A liberti, G. Palmiotti, Nucl. Data 
Sheets, 123, 68-73. (2015)

9. G. Palmiotti, M. Salvatores et al., ND2016, Bruges (2016)

10. Interm. Report of the W PEC Subgroup 33 NEA /NSC/ WPEC/ DOC, 429, (2010)

11. G. Palmiotti and M. Salvatores, A nnals of Nucl. Energy, 52, 10-21, (2013)

12. T. K awano et al., Nucl. Sci. and Eng. Vol. 153, 1-7, (2006)

13. D. Siefman et al., to be published

14. M. A ufiero, et al., $M \& C$ 2017, J eju, K orea, A pril 1620 (2017)

15. Gandini and M. Salvatores, RT/FI(74)3, Comitato per l'Energia Nucleare, Italy (1974).

16. G. Palmiotti, M. Salvatores, H. Hiruta, Trans. A m. Nucl. Society, 104, Hollywood, Florida, J une 26-30, (2011)

17. C. De Saint J ean et al, EPJ W eb of Conferences 146, 02007 (2017). See also C. De Saint J ean et al, Nucl. Data Sheets 123, J anuary 2015, 178-184, and E. Privas, C. De Saint Jean, G. Noguere, EPJ Nuclear Sci. Technol. 4, 36 (2018)

18. G. Palmiotti, et al., J. of the K orean Physical Soc., 59, No. 2, 1123-1128 (2011)

19. G. Palmiotti et al., Nucl. Data Sheets 123, 41-50 (2015)

20. K. Y okoyama, M. Ishikawa, Nucl. Data Sheets, 123 , 97-103 (2015)

21. R. Soule et al., Proc. of the 1988 Intern. Reactor Physics Conference. V olume 2

22. G. Palmiotti et al., Final Report, to be published, OECD/NEA

23. P. Ros, P. Blaise and P. Leconte, Annals of $\mathrm{Nucl}$. Energy 110, 290-305 (2017)

24. P.Ros, P. B laise and P. Leconte, EPJ 146:03007(2017)

25. G. Palmiotti et al., Nucl. Data Sheets 123,41-50. (2015)

26. Intermediate Report, NEA/N SC/R, 6 M ay 2017

27. M. Salvatores, A nnals of Nucl. Energy 36,1653-1662 (2009) 